\title{
Model design of a superconducting quantum interference device of magnetic field sensors for magnetocardiography
}

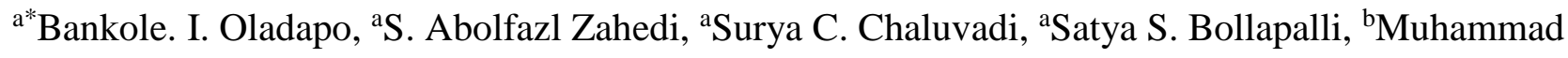 \\ Ismail \\ ${ }^{a}$ School of Engineering and Sustainable Development, De Montfort University, Leicester, UK \\ ${ }^{b}$ Department of Engineering, University of Leicester, Leicester, UK \\ *Corresponding author, Tel: +44(0)1162013971, E-mail: P17243433@my365.dmu.ac.uk
}

\begin{abstract}
In recent years, there has been an increase in the study of magnetocardiography (MCG), complementary to electrocardiography (ECG) research, with the purpose of increasing accuracy in the diagnosis of heart and brain pathologies. This research proposes the physical infrastructure of an advanced technology that can be used to obtain heart and brain signals from a specifically designed magnetic field. A generated magnetic sensor is proposed to sense weak magnetic fields in order to detect magnetic heart and brain activity, using interferometry methods. The method of detection of the magnetic field in the sensor, known as a superconducting quantum interference device (SQUID), is found in the interference that occurs during transmission of feeding currents, and the induced currents in the sensor. The sensor consists of two Josephson junctions, connected in parallel. This research presents a fabrication method and the characteristics of thin superconducting films, as an advance in the construction of a SQUID sensor. An ablation chamber is designed, and the deposition of the superconductor on a copper substrate is explored, to obtain thin films at lower cost. The results obtained show good characteristics of superconductivity which can produce a good quality magnetic sensor. There is an intention to further decrease the roughness of the material for the photolithography process.
\end{abstract}

Keywords: Magnetic field sensor, Magnetocardiography, Electrocardiography, Superconducting quantum interference device

\section{Introduction}

Electrophysiology is a biomedical field dealing with the study of the electrical activity present in cardiac muscle fibre, the ion channels located within the cardiac cell membrane fibre, and its effect on the body. The opening of the various populations of ion channels in cardiac muscle fibre generates electrical currents mediated by various ionic species [1-2]. The electrical coupling between cardiac muscle fibres allows the potential action to propagate through cardiac muscular tissue. Catheter ablation of atrial fibrillation (AF) and detection of the dominant frequency (DF) has evolved rapidly this decade, in both the atria and the coronary sinus (CS). However, in patients with persistent AF, ablation therapy is still challenging because the arrhythmogenic substrate beyond the pulmonary veins (PVs) plays a role in the perpetuation of AF [3]. The electrical signal of the myocardium is the product of the sum of the action potentials of the muscle fibres which propagates passively and diffuses through the body. Because of the passive propagation of electrical signals, only a small part reaches the skin surface, where it can be measured using electrodes, electrocardiography (ECG) or 
magnetocardiography (MCG). In ECG or MCG, the voltage of the signal depends on how the electrodes are placed on the surface of the body and their proximity to the heart or brain [3-6].

The ECG test, however, has many limitations. Firstly, it can only measure cardiac electrical activity indirectly because the true signals from the heart are distorted by elements of electrical resistance that lie between the source of the signal in the heart or brain cells and the ECG electrodes placed on the skin surface. Secondly, the measurements are very directional in nature. Assuming that the ECG electrode is a window through which the heart or brain can be observed, misplaced electrodes give a different view of the heart or brain, and, indeed, a misplaced electrode can change a monophasic wave that is a hill on an ECG trace into a biphasic wave (hills and valleys). As mentioned, the electrical activity of the myocardium is a product of the transmembrane ion currents that occur in cardiac muscle fibres. But, according to the law of Biot-Savart, the charge flow induces a magnetic field, which generates an ionic current in the cardiac muscle fibres. The measurement and recording of these magnetic signals constitute MCG. In summary, the electrical activity of the heart is produced by transmembrane ionic currents. It generates a bioelectrical field detectable by ECG and a bio-magnetic field detectable by MCG.

The instrument for obtaining MCG is more complex than that used for ECG [7]. Because of this complexity, a theoretical and experimental investigation is developed. According to Helmholtz theorem, ECG and MCG are mathematically independent. Therefore, it would be expected that MCG can generate a lot more information than ECG. In practice, the electrical and magnetic signals are detected partially and independently, which reduces the amount of new information that MCG can detect [8]. However, the information that MCG can provide has made it extremely useful. The ECG and MCG statistical information are complementary, but, overall, the human mind is responsible for accurate diagnosis. When both ECG and MCG data are used in analysis and diagnosis, errors in the diagnosis of cardiac pathologies can be reduced by half, compared to using ECG only [9-12]. Additionally, the effectiveness of MCG has been validated in patients with various cardiac abnormalities such as ischemia, cardiomyopathies, atrial and ventricular arrhythmias, etc. Prototypes have been developed that obtain MCG in a stress test, similar to those performed for ECG.

\section{Materials and methods}

MCG conforms to a vectorial relation, which can provide information according to three-dimensional cardiac magnetic activity. It generally takes the component perpendicular to the chest [13-16]. Unlike ECG, MCG has little interaction with the skin, and it is not necessary to have a reference. Similar to the derivations proposed by Eithoven in ECG, Maslennikov et al. propose a $4 \times 4$ mesh which constitutes standard MCG. Fig. 1 shows the recorded measurements of typical MCG of the head taken with a SQUID4 sensor $[1,5]$. 


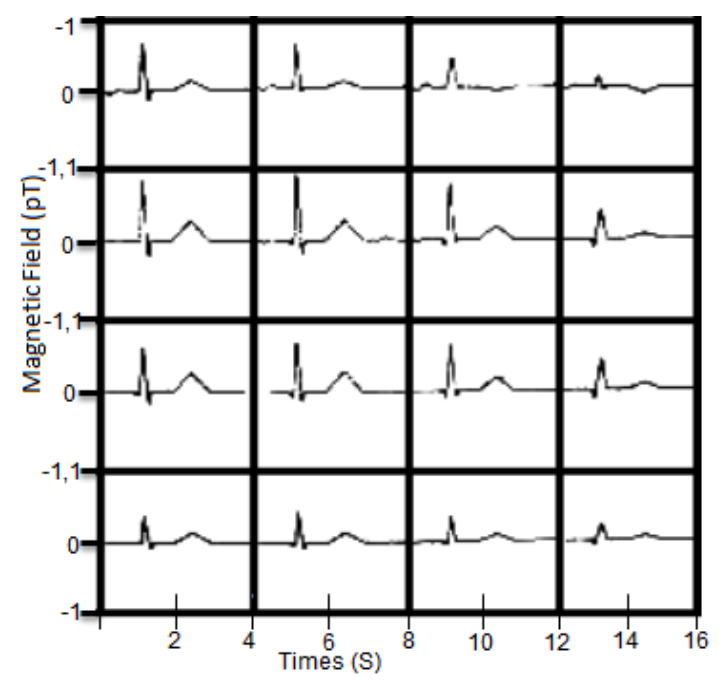

Fig. 1. MCG measurements of magnetic field signal components perpendicular to the plane, according to the mesh proposed by $[1,5]$

Magnetic field measures are the most appropriate technique for measuring extremely weak magnetic fields, such as cardiac fields, and utilize SQUID. This is one the most sensitive devices that exist for MCG [4, 16]. The SQUID16 is an extremely sensitive magnetometer, used to measure extremely subtle magnetic fields based on superconducting loops containing a Josephson junction that converts magnetic flux to voltage. The output voltage is a periodic magnetic flux as follows:

$$
\emptyset_{0}=\frac{h}{2 e}=2.07 \times 10^{-15} \text { weber }(\mathrm{Wb}) \text {. }
$$

Where $\emptyset_{0}$ represents magnetic flux, $h$ is the Plank constant and $e$ is the charge of the electron. In this way, the output voltage detects signals corresponding to a change in the lower flow $\emptyset_{0}$. For its function in SQUID, it combines two physical phenomena: magnetization of magnetic flux; $\varnothing$ magnetic flux in a closed superconducting circuit recast of $\emptyset_{0}$ and the tunnel of the Josephson effect. The direct current (DC) SQUID consists of two Josephson junctions, connected in parallel in a circuit superconductor. It is called a DC SQUID because it operates using a DC current [17-20].

\subsection{SQUID}

This transducer is comprised of two Josephson junctions, co-connected in parallel to form a type of washer. In the arrangement shown in Fig. 2, there is a function wave of common electron pairs at both the top and bottom of the array. For a symmetric, and in the absence of an external, magnetic field arrangement, the phase differences can be seen via the joints. When a charge is applied perpendicular to the magnetic plane of the array a difference in phase arises between the joints. If the critical currents $I_{1}$ and $I_{2}$ are equal, the following expression can be derived [21-23].

$$
I=2 \times I_{2} \times \operatorname{Cos}\left(\frac{\pi \times \emptyset_{\text {ext }}}{\emptyset_{0}}\right) \text {. }
$$

The Josephson junction connections shown in Fig. 2 are structurally parallel to one another. $I$ is the current supply of the system, $\emptyset_{\text {ext }}$ represents the external magnetic flux, and the phase differences of the current through the union is represented by input an output of the Josephson junctions. The constant $\emptyset_{0}$ corresponding to the magnetic flux (equation 1). If we feed the SQUID with a constant current and apply a magnetic flux to the circuit uniformly, the voltage across the SQUID oscillates for a period given by $n \emptyset_{0}$ (with $n=0,1,2, \ldots$ ). If the external field increases slowly, it results in a voltage, the period of which is a multiple of $n \emptyset_{0}$, which makes it possible to measure magnetic fields smaller than a quantum of 
magnetic flux. Fig. 2 shows a SQUID sensor. Despite the advantages of SQUID sensors, the use in MCG for clinical purposes, as in the case of ECG, is limited because it is a subject of research and technological development [6].

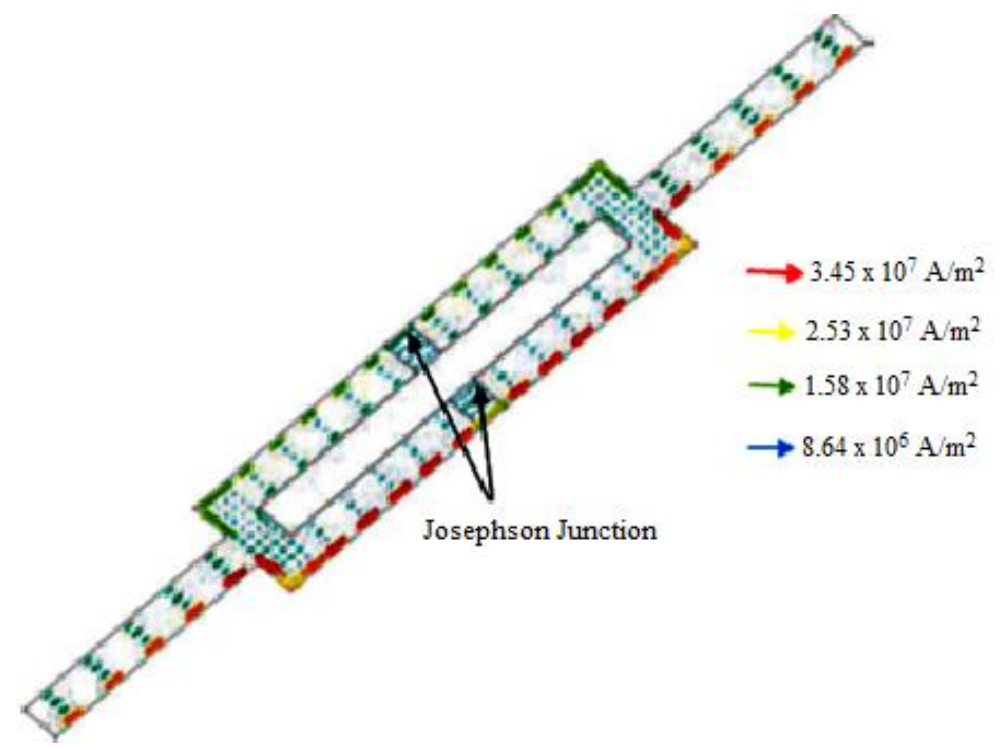

Fig. 2. A force microscope SQUID sensor of an atom of current density distribution in a superconducting state

The measurement of weak magnetic signals, such as those induced by the electrical activity of the heart, requires a technology only available in a few countries [24, 25]. In order to cover this gap in developing countries, a MCG modelling design is proposed in this research. A critical part of obtaining a sensor is the production of appropriate superconducting thin films. In this research, critical model development and characterization of superconducting thin films, as an advance in the construction of weak magnetic field sensors, is proposed. For the growth of thin films, an ablation chamber is designed, modelled, and tested with a low cost substrate.

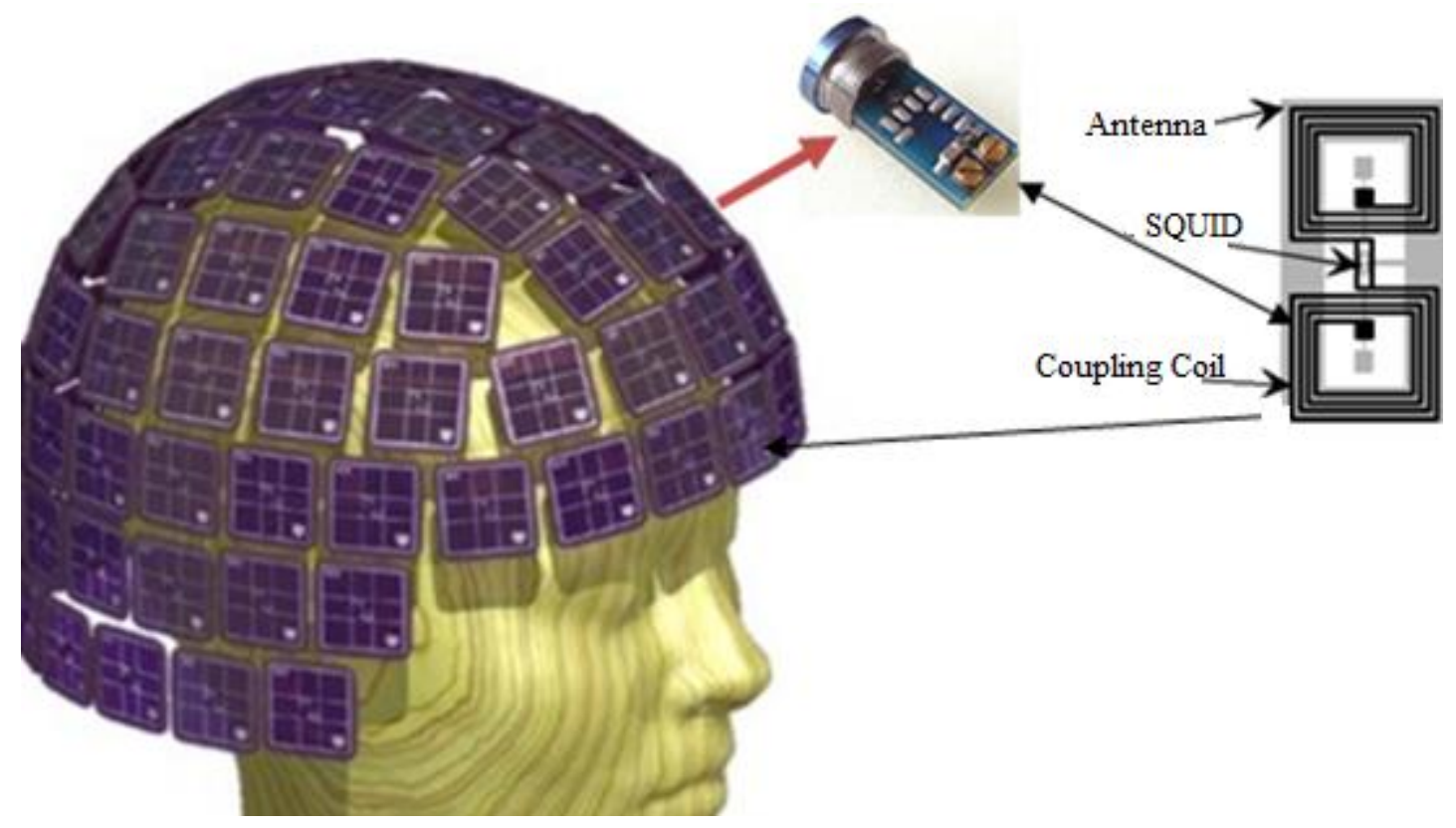

Fig. 3. SQUID sensor and the schematic coupling of the coil in a superconducting state

\subsection{Construction of thin films}


Using the pulsed laser deposition (PLD) method, film is grown on laminated electrolytic copper substrates (with a purity of $99.9 \%$ ), the dimensions of which are $3 \times 3 \mathrm{~cm}$ by $1 \mathrm{~mm}$ thick. This material is suitable for growing this type of film because copper oxide has a monoclinic structure and network parameters very similar to Yttrium Barium Copper Oxide (YBaCuO) [25]. This prevents the film from suffering stresses that can cause its destruction, since the atomic layers that are deposited fit properly to the base of the copper oxide. For the growth of films, the substrate and the target are placed on the supports of the ablation chamber which is designed and modelled in the laboratory. The distance between the laser and the substrate is $3 \mathrm{~cm}$. The vacuum of the chamber is made using a turbomolecular pump, with a pumping speed of 151 litres per second. After reaching a vacuum of $10^{-4} \mathrm{mbar}$ and heating the substrate to $650^{\circ} \mathrm{C}$, the NdYAG laser is applied for 15 minutes creating a plasma that is deposited on the $\mathrm{Cu}$ substrate. The pulse frequency of the laser is $10 \mathrm{~Hz}$ with a pulse width of 5-7 nm and laser pulse $1060 \mathrm{~nm}$. Subsequently, one of the films is subjected to annealing in an oxygen atmosphere at $700^{\circ} \mathrm{C}$ for 4 hours, and the other at $800^{\circ} \mathrm{C}$ for 4 hours [10].

In order to observe the morphology of the surface of the film, images are obtained by scanning electron microscopy and atomic force microscopy. In addition, an X-ray diffraction study is carried out to determine the stoichiometry of the deposited material. The observation of the microstructure and the elemental chemical analyses are carried out in a scanning electron microscope, with a capacity of $40 \mathrm{kV}$, conditioned with a SiLi solid state detector with 1024 channels and a range of $20 \mathrm{kV}$. The micrographs are obtained at an acceleration voltage of $20 \mathrm{kV}$ and a current intensity of $200 \mathrm{pA}$, by means of a secondary electron detector. The chemical analyses are obtained by X-ray diffraction with application of energy dispersion (ED). Also, punctual analysis is performed with an acceleration voltage of $20 \mathrm{kV}$, and current density of $1 \mathrm{nA}$. The acquisition time for each spectrum is 60 seconds in real time. All the elements that generate R-X, from beryllium, are detected. The calculation of the semiquantifications is made using the correction factor ZAF (where $\mathrm{Z}$ is the effect of the atomic number, $\mathrm{A}$ is absorption and $\mathrm{F}$ is the fluorescence of the processed sample), and the results are normalized.

\section{Results and discussion}

The result of the chemical analysis obtained by X-ray diffraction is that the constituent elements of the superconductor Y, $\mathrm{Ba}, \mathrm{O}$ and $\mathrm{Cu}$ are present. The presence of two peaks of $\mathrm{Ba}$ and $\mathrm{Cu}$ is due to ionization. To observe the roughness and smoothness of the material, an image is obtained with the scanning electron microscope. An image of an uncoated sample is shown in Fig. 4. The sample without annealing presents a diffuse granular distribution, as shown in Fig. 4a. An appropriate sample would be one with a larger grain size, since this facilitates electronic transport by decreasing the number of weak junctions. When the sample is annealed, the surface is remarkably modified by increasing the grain size. Fig. 4b shows the image of the sample annealed at $800^{\circ} \mathrm{C}$. Magnetic susceptibility measurements are proposed to investigate the superconducting properties at different magnetic field values of the annealed material. 

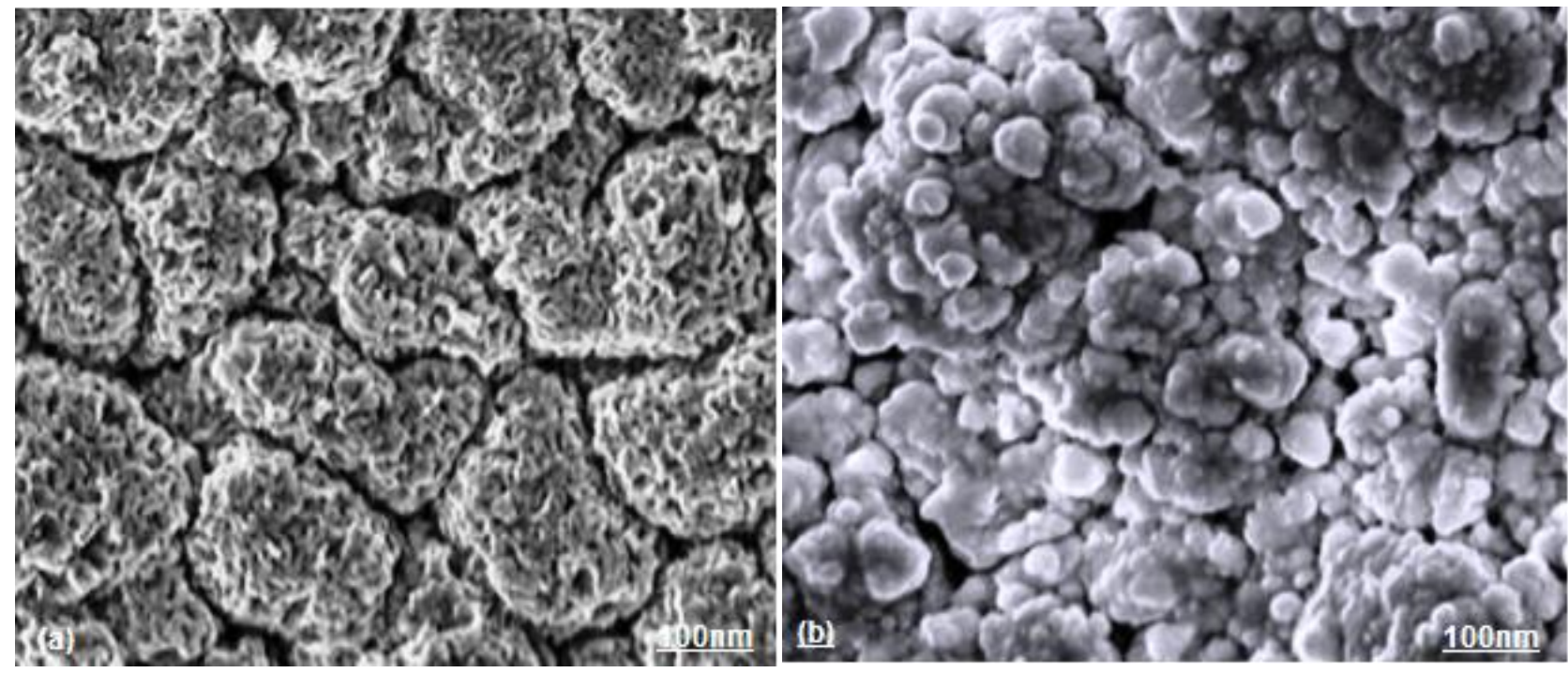

Fig. 4. Images of a thin film using electron microscopy scanning (a) without annealing; (b) annealed at $800^{\circ} \mathrm{C}$

This is done using a closed cycle cryostat, a lock-in, amplifiers and a computer to obtain the data shown in Fig. 5. As shown, the material exhibits superconducting properties, if the transition temperature is close to $40 \mathrm{~K}$. Despite having a fairly large transition width, the films give good results given that the annealing was done in vitro (the method of carrying out a given process in a controlled environment outside a living organism). Fig. $5 \mathrm{~b}$ shows the magnetic susceptibility results of the thin film with an annealing process at $800^{\circ} \mathrm{C}$. The graph shows that the transition temperature increases to approximately $50 \mathrm{~K}$, almost 10 degrees different from the initial temperature.

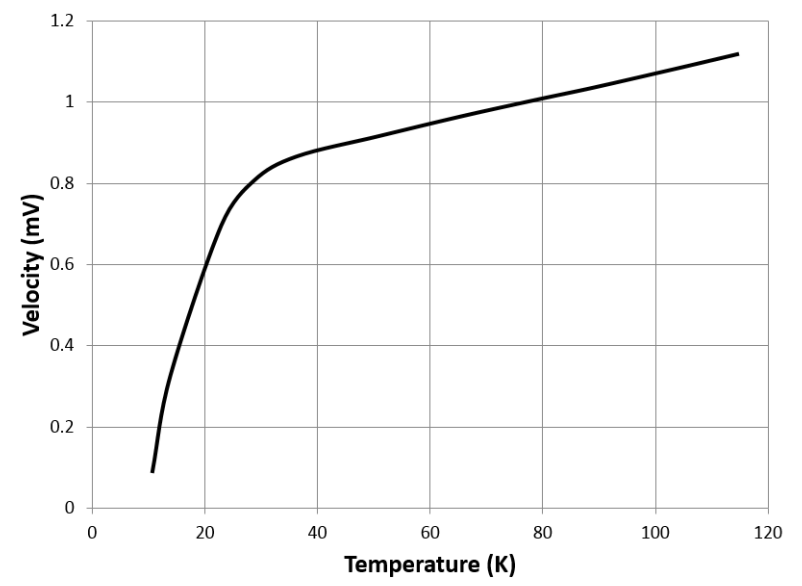

(a)

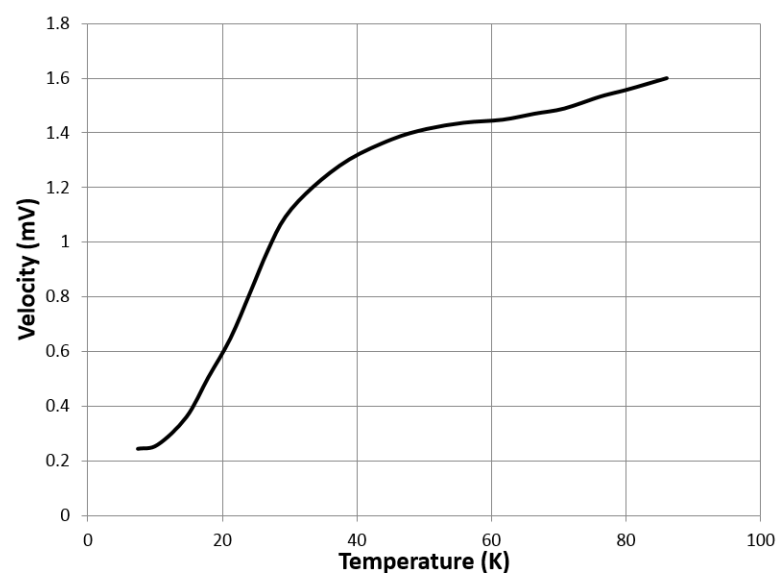

(b)

Fig. 5. Voltage compared to temperature of a thin film for susceptible magnetic films annealed at different temperatures (a) without annealing; (b) annealed

Despite the greater instrumental complexity of MCG with respect to electrocardiography, it has been developed as a valuable complementary technique, generally used by physiologists and cardiologists. Due to its great potential as a tool for the study of cardiac physiology and in the diagnosis of heart disease, it is currently undergoing research. In addition, the development and use of sensors is not limited to the detection of cardiac activity, but also MCG. The development and 
modelling of MCG requires considerable financial and human resources, which has limited its use and research in developing countries. The use of such resources is justified to the extent that MCG increases the possibility of the more accurate and timely diagnosis of heart disease, which is one of the leading causes of death in adults. In this project, an indispensable infrastructure for the growth of thin films of superconducting material with appropriate characteristics for the fabrication of sensors of weak magnetic fields is designed and proposed. We consider this an important step for the production of SQUID, which will bring about the development of MCG. Generally, the growth of thin superconducting films is carried out on substrates of strontium titanate $\left(\mathrm{SrTiO}_{3}\right)$ [22-24]. One important aspect of the present work is proposing a model for the development of a thin film of superconducting material using copper substrates, which will reduce the cost of SQUID sensors. A strontium titanate substrate costs around 100 times more than copper one. Apart from the cost effectiveness of copper, it has approximately $25 \%$ higher thermal conductivity than strontium titanate $\left(\mathrm{SrTiO}_{3}\right)$ [24]. It has superior electrical and thermal conductivity, greater dependability of bonding at higher operating temperatures, less intimate growth, and higher mechanical stability. SrTiO3 has a space group of 3.5-4.5 A, a lattice parameter equal to $0.39059 \mathrm{~A}$ at a relative permittivity of around $300 \mathrm{~K}$, and a dielectric loss of $\tan \delta$ (ratio of the imaginary and real parts of the permittivity) at $10 \mathrm{GHz}$ of around $5 \times 10^{-4}$ in $300 \mathrm{~K}$ to $3 \times 10^{-4}$ in $77 \mathrm{~K}$, and a thermal expansion coefficient of $9.4 \times$ $10^{-6} \mathrm{~K}^{-1}$. On the other hand copper has a greater lattice parameter 3.61 A and a thermal expansion coefficient of $6.8 \times$ $10^{-6} \mathrm{~K}^{-1}$. However, $\mathrm{SrTiO}_{3}$ has high dielectric loss and excessively high dielectric constant of electric field bias, relative permittivity at $300 \mathrm{~K}$ which makes it less suitable as a substrate material $[26,27]$. The results indicate that the films have good superconducting characteristics, which means that it is possible to build magnetic sensors of good quality. However, before building sensors, it is possible to make improvements to the films. The practical realization of the sensor requires thin film growth techniques as well as photolithography techniques [17]. Therefore, it is intended to further reduce the roughness of the material, so the photolithography process is carried out under optimal conditions, and, in this way, the appropriate geometry of the SQUID is obtained. The next stage of the project is to increase the critical temperature and decrease the roughness. Therefore, we are currently working on the process of growing thin films in an oxygen controlled environment by adding this gas to the ablation system.

\section{Conclusion}

In this research, a method of production of a critical part of a magnetic sensor to sense weak magnetic fields in order to detect heart and brain activity using interferometric methods is proposed. The magnetic field is detected as a function of the interference between the feeding and induced currents in the well-known SQUID sensor, which consists of two Josephson junctions connected in parallel. The characteristics of superconducting thin films used in the construction of SQUID sensors, for which sensor built ablation is introduced, are explored. The deposition of a superconductor on a substrate of $\mathrm{Cu}$ is proposed. MCG can detect more in the DF, and CS than ECG. However, the usefulness of pre-procedural detection of the AF frequency gradient for ablation therapy needs to be evaluated in future studies.

\section{Acknowledgment}

This project is funded by the Higher Education Innovation Fund (HEIF) of De Montfort University 2017-2018, UK: Research Project No. 0042.07. 


\section{References}

[1] Y.V. Maslennikov, M.A. Primin, V.Y. Slobodchikov, V.V. Khanin, I.V. Nedayvoda, V.A. Krymov, A.V. Okunev, E.A. Moiseenko, A.V. Beljaev, V.S. Rybkin, A.V. Tolcheev, A.V. Gapelyuk, The DC-SQUID-based magnetocardiographic systems for clinical use, Physics Procedia 36 (2012) 88 - 93.

[2] L.D. Tung, J. Schaefer, M.R. Lees, G. Balakrishnan, D.M. Paul, Magnetic properties of a LuVO3 single crystal studied by magnetometry, heat capacity and neutron diffraction, Journal of science: advanced materials and devices, 1 (2016) 174-178.

[3] K. Yoshida, K. Ogata, T. Inaba, Y. Nakazawa, Y. Ito, I. Yamaguchi, A. Kandori, K. Aonuma, Ability of MCG to detect regional dominant frequencies of atrial fibrillation, Journal of arrhythmia, 31 (2015) 345-351.

[4] X. Kong, S. Zhang, J. Wang, J. Zeng, X. Xie, Multi-channel magnetocardiogardiography system based on low-Tc SQUIDs in an unshielded environment, Physics procedia 36 (2012) 286 - 292.

[5] J. Malmivuo, R. Plonsey. Bioelectromagnetism principles and applications of bioelectromagnetic fields. Oxford University press, New York. 1995

[6] J.R. Immanuel, V. Prabhu, J.V. Christopheraj, D. Sugumar, T. Vanathi, Separation of maternal and fetal ECG signals from the mixed source signal using FASTICA, Procedia engineering 30 (2012) $356-363$.

[7] M.A. Hasan, M.B.I. Reaz, Detection and processing techniques of FECG signal for fetal monitoring, Biological procedures online, 11(1) (2009) 263-295.

[8] M. Kania, M. Fereniec, Wavelet denoising for multi-lead high resolution ECG signals, Measurement science review 7(2) (2007) 30-33.

[9] S.L. Zhang, Y.L. Wang, H.W. Wang, S.Q. Jiang, X.M. Xie, Quantitative evaluation of signal integrity for magnetocardiography, Physical medical biology 54 (2009) 4793-4802.

[10] A.O.M. Adeoye, B.I. Oladapo, A.A. Adekunle, A.J. Olademeji, J.F.Kayode, Design, simulation and implementation of a PID vector control for EHVPMSM for an automobile with hybrid technology. Journal of materials research and technolog, https://doi.org/10.1016/j.jmrt.2017.07.005

[11] G. Yang, Z. Zeng, Y. Deng, L. Udpa, S.S. Udpa, 3D EC-GMR sensor system for detection of subsurface defects at steel fastener sites, NDT \& E International, 50 (2012) 20-28.

[12] G.R. Helena, K. Luka, A.L. Ribeiro, Characterization of a straight crack by performing measurements in two different directions, AIP conference proceeding, 1335 (2011) 673-680.

[13] A.O.M. Adeoye, J.F. Kayode, B.I .Oladapo, S.O. Afolabi, Experimental analysis and optimization of synthesized magnetic nanoparticles coated with PMAMPC-MNPs for bioengineering application, St. petersburg polytechnical university journal: physics and mathematics, 3 (4) (2017) 333-338

[14] D. Pasadas, T. R. Jorge, H.H. Ramos, A.R. Lopes, Evaluation of portable ECT instruments with positioning capability, Measurement, 45(3) (2012) 393-404.

[15] V.A. Baloguna, B.I. Oladapo, A.O.M. Adeoye, J.F. Kayode, S.O. Afolabi, Hysteresis analysis of Thornton (IP6, IP12E and $\mathrm{TH} 5 \mathrm{~V}$ ) magnetic materials through the use of arduino microcontroller, Journal of materials research and technology, https://doi.org/10.1016/j.jmrt.2017.05.018.

[16] M.R. Djamal, Development of sensors based on giant magnetoresistance material. Procedia enginering, 32 (2012) 60 -68 . 
[17] A. Corsini, C. Tortora, Unsteady end-wall pressure measurements using near-field DIY sensors on fouled fan rotor blades, Energy procedia, 82 (2015) 237 - 244.

[18] S. Bianchi, A. Corsini, A.G. Sheard, C. Tortora, A Critical review of stall control techniques in industrial fans, International scholary research notice mechanical engineering, 52 (6) (2013) 192-197.

[19] L. Bian, Y. Wen, P. Li, Y. Wu, X. Zhang, M. Li, Magnetostrictive stress induced frequency shift in resonator for magnetic field sensor, Sensors and Actuators A, 247 (2016) 453-458.

[20] B. Bergmair, T. Huber, F. Bruckner, C. Vogler, D. Suess, Removal of earth's magnetic field effect on magnetoelastic resonance sensors by an antisymmetric bias field, Sensors and actuators A, 183 (2012) 11- 15.

[21] C.A. Grimes, S.C. Roy, S. Rani, Q. Cai, Theory, instrumentation and applications of magnetoelastic resonance sensors: a review, Sensors, 11(3) (2011) 2809-2844.

[22] K. Ong, K. Zeng, X, Yang, K. Shankar, C. Ruan, C. Grimes, Quantification of multiple bioagents with wireless, remote-query magnetoelastic microsensors, IEEE Sensors J., 6 (3) (2006) 14-522.

[23] B.I.Oladapo, A.O.M. Adeoye, S.O. Oyegoke, S.O.Afolabi, Analysis for distortion of thin-wall milling on machine component and its effect on global warming, Procedia manufacturing, 7 (2017), 529-536

[24] D. Suess, R. Groessinger, Sensor device for contactless measuring of temperatures by using magnetic materials in the vicinity of phase crossovers, European Patent, (2011) 228-262.

[25] D.J. Pasadas, T.J. Rocha, H.G. Ramos, A.L. Ribeiro, Remote field eddy current inspection of metallic tubes using GMR sensors, Proceeding of the IEEE instrumentation and measurement technology conference, Minnesota, MN, EUA (2013).

[26] P. Bao, T.J. Jackson, X. Wang, M.J. Lancaster, Barium strontium titanate thin film varactors for room-temperature microwave device applications, Journal of physics D: applied physics, 41 (2008) 063001 1-21

[27] D J. Keeble, S. McGuire, S. Singh, B. Su, TW. Button, J. Petzelt, Journal of physics. IV (France), 128 (2005) 111 